\title{
The Role of Maintenance Therapy in the Treatment of Multiple Myeloma
}

Ashraf Z. Badros, MD, Baltimore, Maryland

\author{
Key Words \\ Autologous stem cell transplantation, interferon, thalidomide, \\ bortezomib, lenalidomide
}

\begin{abstract}
Maintenance therapy in multiple myeloma has been under investigation for more than 3 decades, without evidence of clear benefit until recently. Chemotherapy maintenance offers no benefit after conventional or high-dose treatment. Interferon-based maintenance is associated with minimal improvements in clinical outcomes, but is poorly tolerated. Results of corticosteroid maintenance studies have been conflicting; at least one randomized trial showed improved survival with prednisone maintenance after conventional chemotherapy. The role of the novel agents thalidomide, lenalidomide, and bortezomib as maintenance is emerging. Most reported maintenance studies have evaluated thalidomide, alone or in combination with a corticosteroid. Several of these studies suggest that thalidomide-based maintenance prolongs overall survival after autologous stem cell transplantation. Important questions that have not yet been resolved include the optimal dose and duration of thalidomide, whether clinical benefit depends on response to induction therapy and risk for relapse, and whether reported benefits are caused by cytoreduction or eradication of minimal residual disease, especially with bortezomib maintenance. Ongoing randomized trials are evaluating lenalidomide and bortezomib maintenance therapies to better define the role of these drugs as maintenance in multiple myeloma. (JNCCN 2010;8[Suppl 1]:S21-S27)
\end{abstract}

Maintenance therapy in multiple myeloma (MM) comprises any therapy given "following completion of induction treatment in responding or nonprogressing patients, with the goal of prolonging survival." ${ }^{\text {Howev- }}$

From the Bone Marrow Transplant Program, Greenebaum Cancer Center, University of Maryland, Baltimore, Maryland.

This work was supported by an educational grant from Millennium Pharmaceuticals, Inc.

Correspondence: Ashraf Z. Badros, MD, University of Maryland,

Greenebaum Cancer Center, Bone Marrow Transplant Program, 22

South Greene Street, Baltimore, MD 21201.

E-mail: abadros@maryland.umaryland.edu er, maintenance therapy practice patterns vary considerably because of conflicting data about its effectiveness. Currently, NCCN recommends thalidomide (category 1 recommendation) with or without prednisone (category 2B) for maintenance therapy; the roles of interferon and corticosteroids are less clear (category 2B). ${ }^{2}$ This article summarizes the results of many clinical trials that have been designed to evaluate maintenance after conventional chemotherapy and high-dose therapy and identifies areas of current controversy in an effort to understand the role of maintenance therapy in managing patients with newly diagnosed MM.

\section{Maintenance After Conventional Chemotherapy}

Because of the increased risk for acute myeloid leukemia with prolonged alkylating agent therapy, alternatives to this approach were being sought as early as the 1980s. Interferon-alpha was the subject of numerous trials that produced inconclusive results. Two meta-analyses, one performed using individual patient data and the other using published data, found a small but statistically significant survival benefit when interferon maintenance was used after conventional therapy. ${ }^{3,4}$ However, few patients can tolerate the substantial toxicities of interferon therapy, and therefore interferon maintenance is rarely used.

Several studies have evaluated corticosteroids, with or without interferon, as maintenance, but the data are insufficient to draw firm conclusions. When compared directly with interferon maintenance, dexamethasone, $20 \mathrm{mg} / \mathrm{m}^{2}$, given orally on days 1 to 4 of a 28 -day cycle produced a similar median duration of remission, but significantly fewer patients treated with dexamethasone maintenance responded to repeat melphalan/dexamethasone at relapse relative to those treated with in- 
terferon maintenance $(44 \%$ vs. $82 \% ; P=.001) .{ }^{5}$ In the MY.7 study by the National Cancer Institute of Canada (NCIC), dexamethasone maintenance improved median progression-free survival (PFS) based on a hazard ratio (HR) of $0.61(95 \% \mathrm{CI}, 0.47-0.79)$, but not overall survival (OS; HR, 0.88; 95\% CI, $0.65-1.18)$, relative to no maintenance. ${ }^{6}$ Similarly, when added to interferon, thrice-weekly prednisone, $50 \mathrm{mg}$, improved median PFS but not overall survival (OS), relative to interferon alone in the SWOG 9028 trial. $^{7}$

Only one trial, SWOG 9210, has shown a survival benefit with corticosteroid maintenance therapy. ${ }^{8}$ In this randomized trial, a prednisone regimen of $50 \mathrm{mg}$ every other day significantly improved median PFS (14 vs. 5 months; $P=.003$ ) and OS (37 vs. 26 months; $P=.05)$ relative to a physiologic dose regimen (10 mg every other day). These data support the use of steroids in this setting, although the efficacy of the SWOG 9210 regimen has not been confirmed in another controlled clinical trial.

Recently, several trials have focused on the role of novel agents as maintenance therapy. Offidani et al. ${ }^{9}$ conducted a randomized comparison of maintenance with either thalidomide $(100 \mathrm{mg} / \mathrm{d})$ plus dexamethasone ( $20 \mathrm{mg} / \mathrm{d}$ for 4 days per month) or interferon thrice weekly plus dexamethasone in 103 patients who experienced response to induction therapy with ThaDD (thalidomide, dexamethasone, and pegylated liposomal doxorubicin). Both 2-year PFS and OS rates were significantly better with thalidomide-based maintenance (PFS, $63 \%$ vs. 32\%; $P=.024$; OS, $84 \%$ vs. $68 \% ; P=.030)$. The low-dose thalidomide regimen was generally well-tolerated, with peripheral neuropathy $(6 \%)$ and constipation (4\%) the most commonly reported grade $3 / 4$ toxicities. More patients were compliant with thalidomide therapy long-term relative to interferon.

Preliminary data have been reported for maintenance therapy in elderly patients who experienced stable disease or better with thalidomide/dexamethasone or melphalan/prednisone induction. ${ }^{10} \mathrm{~A}$ total of 111 patients with nonprogressive disease were randomized to maintenance with thalidomide, 100 mg daily, with thrice-weekly interferon or interferon alone. At a median follow-up of 17.2 months, median survival from the start of maintenance was 53.1 months in the thalidomide/interferon arm and not yet reached in the interferon-alone arm $(P=.49)$.
Data on bortezomib-based maintenance strategies are beginning to emerge. A randomized phase III trial conducted by the Italian Multiple Myeloma Group is comparing bortezomib/melphalan/prednisone/thalidomide (VMPT) induction followed by bortezomib/thalidomide maintenance to bortezo$\mathrm{mib} / \mathrm{melphalan} /$ prednisone (VMP) induction without maintenance in elderly patients with newly diagnosed myeloma. ${ }^{11}$ Results presented at the 2008 American Society of Hematology Annual Meeting showed a higher response rate for VMPT relative to VMP (complete response [CR] rate, $31 \%$ vs. $16 \%$; $P=.003$; very good partial response [VGPR] rate, $55 \%$ vs. $42 \% ; P=.02$ ) but no difference in OS at 3 years.

\section{Maintenance After Autologous Stem Cell Transplantation}

Chemotherapy and Interferon Maintenance After Autologous Stem Cell Transplantation

As with conventional induction regimens, chemotherapy is not currently recommended as a maintenance strategy after autologous stem cell transplantation (ASCT). ${ }^{2}$ Interferon is currently considered a maintenance option in the NCCN Clinical Practice Guidelines in Oncology for Multiple Myeloma (to view the most recent version of these guidelines, visit the NCCN Web site at www.NCCN.org), based on low-level evidence and non-uniform NCCN consensus (category 2B). ${ }^{2}$ However, given the lack of clear activity and substantial toxicity associated with interferon and the availability of new agents, research into interferon maintenance has been largely abandoned.

\section{Novel Agents After ASCT}

Thalidomide is the best studied of the novel agents in the post-ASCT maintenance setting, although randomized clinical trials of bortezomib and lenalidomide are underway (Table 1). ${ }^{12}$ Four randomized phase III trials have been completed to establish the role of thalidomide-based maintenance after ASCT (Table 2). ${ }^{13-16}$ In all 4 studies, improvements in response (CR) and PFS were seen. Of the 4 trials, 3 also reported significant improvements in OS, and a meta-analysis of these data showed a trend toward improved survival with maintenance thalidomide after ASCT (Figure 1). ${ }^{17}$ When the trial that in- 
Maintenance Therapy in Multiple Myeloma

\begin{tabular}{|c|c|c|c|}
\hline Clinical Trial and Accrual Goal & Primary Treatment & Maintenance Regimen(s) & $\begin{array}{l}\text { Primary End } \\
\text { Point }\end{array}$ \\
\hline $\begin{array}{l}\text { NCIC MY.10 } \\
(\mathrm{N}=324) \\
{[\mathrm{NCT} 00049673]}\end{array}$ & ASCT & $\begin{array}{l}\text { - Thalidomide daily with alternate-day } \\
\text { prednisone } \\
\text { - Observation } \\
\text { Maintenance } \times 4 \text { y }\end{array}$ & OS \\
\hline $\begin{array}{l}\text { NHLBI/ BMTCTN0102 }(\mathrm{N}=710) \\
\text { [NCT00075829] }\end{array}$ & $\begin{array}{l}\text { Tandem transplantation } \\
\text { (no HLA-matched sibling) } \\
\text { or ASCT followed by } \\
\text { mini-allogeneic SCT (HLA- } \\
\text { matched sibling) }\end{array}$ & $\begin{array}{l}\text { - Thalidomide/dexamethasone } \\
\text { - Observation } \\
\text { Maintenance } \times 1 \text { y; only administered to } \\
\text { patients undergoing tandem transplantation }\end{array}$ & 3-y PFS \\
\hline $\begin{array}{l}\text { CALGB } 100104 \\
(\mathrm{~N}=462) \\
{[\mathrm{NCT} 00114101]}\end{array}$ & ASCT & $\begin{array}{l}\text { - Lenalidomide } \\
\text { - Placebo } \\
\text { Maintenance continues as tolerated or until } \\
\text { progression; only administered to patients } \\
\text { with stable or responding disease after ASCT }\end{array}$ & TTP \\
\hline $\begin{array}{l}\text { IFM 2005-02 } \\
(\mathrm{N}=614) \\
{[\mathrm{NCT} 00430365]}\end{array}$ & ASCT & $\begin{array}{l}\text { - Lenalidomide } \\
\text { - Placebo } \\
\text { Duration not specified }\end{array}$ & $\begin{array}{l}\text { Duration of } \\
\text { post-ASCT } \\
\text { response }\end{array}$ \\
\hline $\begin{array}{l}\text { Gesellschaft für Medizinische } \\
\text { Innovation } \\
(\mathrm{N}=194) \\
\text { [NCT00891384] }\end{array}$ & $\begin{array}{l}\text { ASCT } \\
\text { Consolidation: } \\
\text { lenalidomide, } 25 \mathrm{mg} \text {, days } \\
1-21 \mathrm{q} 4 \mathrm{w} \times 6\end{array}$ & $\begin{array}{l}\text { - Lenalidomide, } 5 \mathrm{mg} \text {, days } 1-21 \mathrm{q} 4 \mathrm{w} \\
\text { - Lenalidomide, } 25 \mathrm{mg} / \mathrm{d} \text {, days } 1-21 \mathrm{q} 4 \mathrm{w} \\
\text { Maintenance continues until disease } \\
\text { progression }\end{array}$ & EFS \\
\hline $\begin{array}{l}\text { HOVON-65/GMMG-HD4 } \\
(\mathrm{N}=825)^{12}\end{array}$ & $\begin{array}{l}\text { Induction: VAD vs. PAD } \\
\text { Stem cell collection: CAD } \\
\text { ASCT: HDM }\end{array}$ & $\begin{array}{l}\text { - Thalidomide } 50 \mathrm{mg} / \mathrm{d} \\
\text { - Bortezomib } 1.3 \mathrm{mg} / \mathrm{m}^{2} \mathrm{q} 2 \mathrm{w} \\
\text { Maintenance } \times 2 \mathrm{y}\end{array}$ & PFS \\
\hline $\begin{array}{l}\text { PETHEMA GEM05 } \\
(\mathrm{N}=390) \\
{[\mathrm{NCT} 00461747]}\end{array}$ & $\begin{array}{l}\text { Induction: VBMCP-VBAD- } \\
\text { bortezomib vs. TD vs. VTD } \\
\text { followed by ASCT }\end{array}$ & $\begin{array}{l}\text { - Interferon } \\
\text { - Thalidomide } \\
\text { - Thalidomide plus bortezomib } \\
\text { Maintenance } \times 3 \text { y }\end{array}$ & Not stated \\
\hline
\end{tabular}

Abbreviations: ASCT, autologous stem cell transplantation; CAD, cyclophosphamide/doxorubicin/dexamethasone; EFS, eventfree survival; HDM, high-dose melphalan; HLA, histocompatibility leukocyte antigen; OS, overall survival; PAD, bortezomib/ doxorubicin/dexamethasone; PFS, progression-free survival; SCT, stem cell transplantation; TD, thalidomide/dexamethasone; TTP, time-to-progression; VAD, vincristine/doxorubicin/dexamethasone; VBMCP-VBAD, vincristine/carmustine/melphalan/ cyclophosphamide/prednisone alternating with vincristine/carmustine/doxorubicin/dexamethasone; VTD, bortezomib/ thalidomide/dexamethasone.

cluded thalidomide during induction was excluded, the OS benefit became significant (HR, 0.49; 95\% CI, 0.32-0.74)..$^{17}$ Peripheral neuropathy complicated the treatment course in all 4 studies, and, unsurprisingly, the incidence was highest when the duration of thalidomide maintenance was prolonged. None of the studies, however, determined the optimal dose or duration of thalidomide-based maintenance after ASCT.

A recent update to one of the trials, conducted at a median follow-up of 6 years, suggests that thalidomide may benefit patients with high-risk myeloma. ${ }^{18}$ The estimated OS rate at 5 years was $56 \%$ for patients with cytogenetic abnormalities treated with thalidomide versus $43 \%$ for those with cytogenetic abnormalities in the control group $(P=.02)$.

Results from other randomized trials of thalidomide maintenance were presented at national meetings within the past year, none of which supports an improvement in OS. The United Kingdom Medical Research Council (MRC) Myeloma IX study evaluated thalidomide maintenance after primary treatment of myeloma (either ASCT or conventional chemotherapy, depending on the clinical situation). ${ }^{19}$ A total of 820 patients were randomly assigned to thalidomide maintenance $(100 \mathrm{mg} / \mathrm{d}$ until relapse) or no maintenance, making this the largest trial conducted. Overall, PFS was numerically higher in the thalidomide arm, but the improvement was statistically significant only among patients who ex- 
Badros

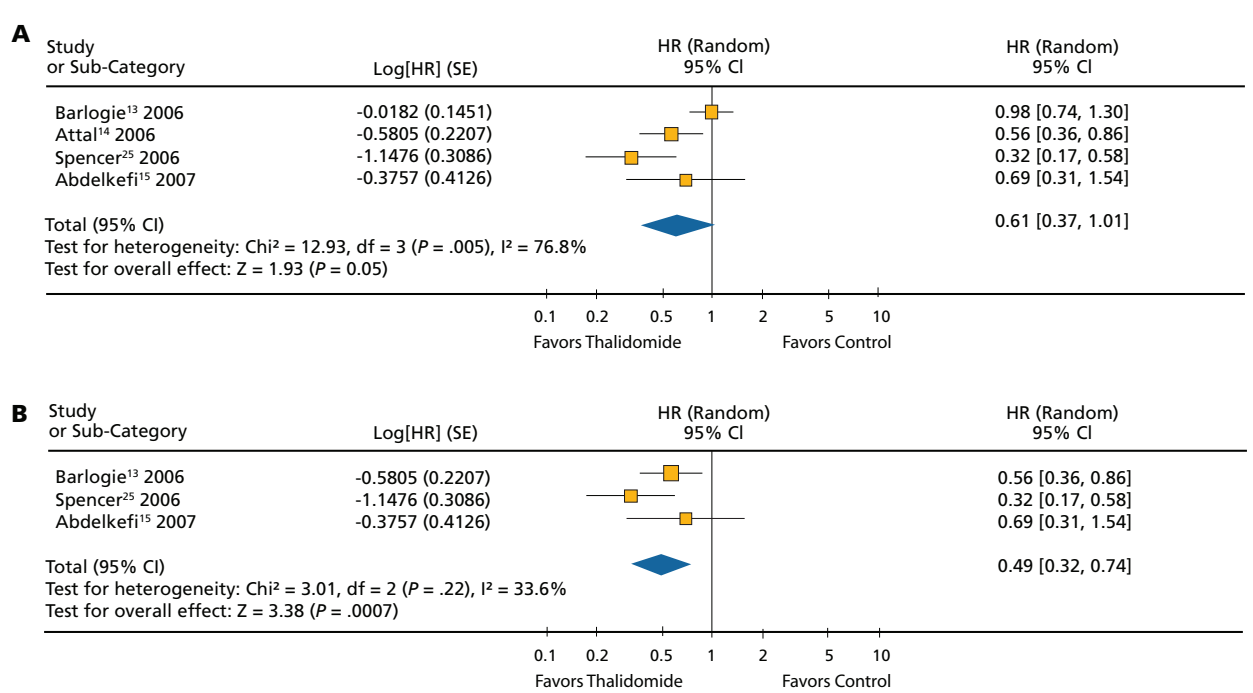

Figure 1 Overall survival with maintenance thalidomide. (A) All trials. (B) Excluding Barlogie 2006.

Abbreviations: HR, hazard ratio; OS, overall survival; SE, standard error.

Adapted from Hicks LK, Haynes AE, Reece DE, et al. A meta-analysis and systematic review of thalidomide for patients with previously untreated multiple myeloma. Cancer Treat Rev 2008;34:442-452, with permission from Elsevier.

perienced less than a VGPR with induction therapy (HR, $1.9 ; \mathrm{P}=.007)$. OS was not significantly improved in any subgroup. Moreover, subgroup analyses showed that patients with $17 \mathrm{p}$ deletions had a significantly worse $\mathrm{OS}$ when treated with thalidomide maintenance ( $\mathrm{HR}, 4.55 ; P=.02$ ), leading the researchers to conclude that $17 \mathrm{p}$ deletion is a contraindication to thalidomide maintenance.

Two small studies presented at the XII International Myeloma Workshop in 2009 showed significant improvements in PFS, but not OS, with thalidomide-based maintenance. Mounier et al. ${ }^{20}$ compared an intermittent dexamethasone/thalidomide maintenance regimen with no maintenance after ASCT in 108 patients and observed median event-free survival of 32 versus 17 months, respectively $(P=.02)$. Maiolino et al. ${ }^{21}$ compared dexamethasone maintenance with dexamethasone plus thalidomide, $200 \mathrm{mg}$ daily, after ASCT in 212 patients. Thalidomide significantly reduced the risk for progression relative to dexamethasone alone (HR, $2.43 ; P=.03)$, but OS was similar in the 2 arms $(65 \%$ vs. $74 \%$ with dexamethasone and dexamethasone/ thalidomide, respectively).

Therefore, although data support a role for thalidomide-based maintenance therapy after ASCT, several important questions remain, such as whether thalidomide given post-transplantation truly maintains response versus early initiation of salvage therapy. Two large studies have reported that the survival benefits with thalidomide were limited to patients who did not experience at least a VGPR, supporting the hypothesis that post-transplantation thalidomide provides additional cytoreduction rather than eradication or suppression of minimal residual disease. Currently, however, initial response to therapy should not be used to guide maintenance treatment decisions.

Furthermore, the optimal dose and duration of maintenance thalidomide remains to be determined. The incidence of neuropathy is clearly cumulative and dose-related, partly leading to the recommendation that thalidomide not be used throughout the entire treatment course. Doses of $200 \mathrm{mg}$ daily or more are difficult to administer on a long-term basis. $^{22,23}$ Lower-dose $(100 \mathrm{mg} / \mathrm{d})$ and time-limited therapy (6-12 months) were efficacious in studies by the Tunisian Multiple Myeloma Study Group (TMMSG) and Australasian Leukaemia and Lymphoma Group (ALLG), but the United Kingdom MRC Myeloma IX study did not show a survival benefit with a 100 mg daily thalidomide maintenance regimen.

Another intriguing issue is whether patients who begin maintenance treatment with less than a VGPR should discontinue therapy once they experience VGPR or CR, given that those with VGPR/CR before maintenance therapy did not seem to benefit from additional therapy in some trials. The NCCN 
Maintenance Therapy in Multiple Myeloma

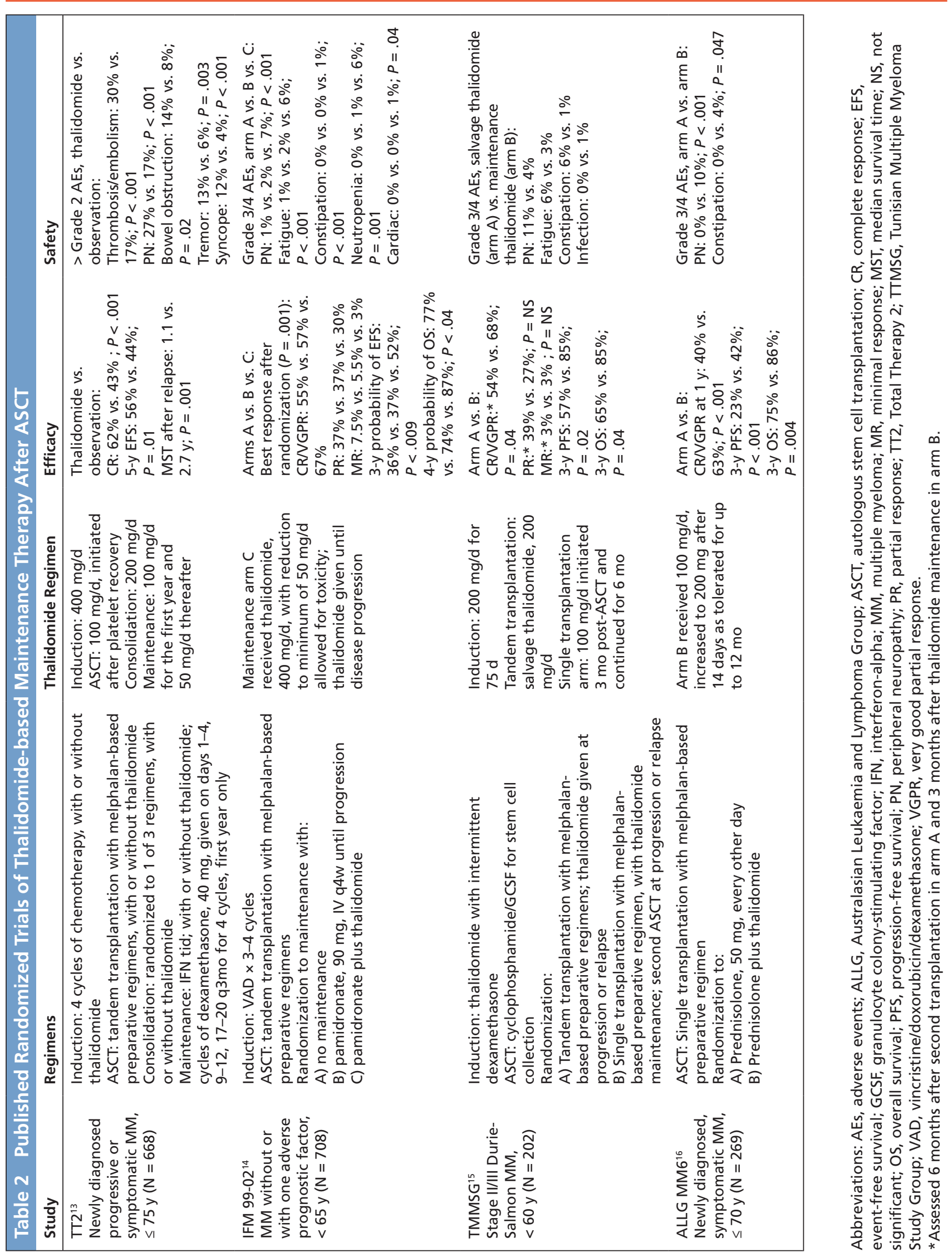


guidelines currently do not address the dose or duration of maintenance thalidomide. Most centers currently continue patients on thalidomide for 1 to 2 years, depending on the patient's tolerance.

Finally, more research is needed to determine whether cytogenetics can be used to identify appropriate candidates for post-transplantation maintenance. Subgroup analyses from Total Therapy 2 suggest that thalidomide maintenance reduced the risk for death in patients with metaphase cytogenetic abnormalities, a well-known adverse prognostic factor, but not in those without these abnormalities. ${ }^{18}$ The IFM 99-02 trial showed that thalidomide was not effective in patients with del(13), and the United Kingdom MRC Myeloma IX trial showed that risk for death was significantly increased with thalidomide maintenance in patients with del (17p). ${ }^{14}$

As these authors describe elsewhere in this supplement, increasing evidence suggests that the novel agents bortezomib and lenalidomide may overcome the poor prognosis associated with cytogenetic abnormalities in myeloma. ${ }^{24}$ How these findings from the induction setting translate into the maintenance setting remains to be determined in appropriately designed clinical trials.

\section{Conclusions}

The role of maintenance therapy after conventional chemotherapy remains to be determined, but there is a paucity of ongoing research in this setting. Much work is needed to evaluate maintenance therapy after ASCT, yet the optimal treatment regimen has not been identified. Thalidomide is the first novel agent to be studied as a maintenance therapy and shows the most promise. However, it may work through a direct cytoreductive effect rather than a true maintenance effect, given that several trials showed benefit only in patients who had not experienced at least a VGPR before initiating maintenance treatment. The optimal dose and duration of thalidomide maintenance remains to be determined, as does the appropriate patient population for routine treatment. Moreover, whether thalidomide should be used as maintenance or at relapse remains a matter of debate. More robust results of trials with lenalidomide and bortezomib maintenance therapies are eagerly awaited.

\section{Acknowledgment}

The author thanks Lori Keys Pender, MPH, BCOP, for medical writing assistance.

\section{References}

1. Anderson KC, Kyle RA, Rajkumar SV, et al. Clinically relevant endpoints and new drug approvals for myeloma. Leukemia 2008;22:231-239.

2. Anderson KC, Alsina M, Bensinger W, et al. NCCN clinical practice guidelines in oncology: multiple myeloma. Version 2, 2010. Available at: http://www.nccn.org/professionals/physician_ gls/PDF/myeloma.pdf. Accessed May 19, 2009.

3. Fritz E, Ludwig H. Interferon-alpha treatment in multiple myeloma: meta-analysis of 30 randomised trials among 3948 patients. Ann Oncol 2000;11:1427-1436.

4. Myeloma Trialists' Collaborative Group. Interferon as therapy for multiple myeloma: an individual patient data overview of 24 randomized trials and 4012 patients. Br J Haematol 2001;113:10201034 .

5. Alexanian R, Weber D, Dimopoulos M, et al. Randomized trial of alpha-interferon or dexamethasone as maintenance treatment of multiple myeloma. Am J Hematol 2000;65:204-209.

6. Shustik C, Belch A, Robinson S, et al. A randomized comparison of melphalan with prednisone or dexamethasone as induction therapy and dexamethasone or observation as maintenance therapy in multiple myeloma: NCIC CTG MY.7. Br J Haematol 2006;136:203-211.

7. Salmon SE, Crowley JJ, Balcerzak SP, et al. Interferon versus interferon plus prednisone remission maintenance therapy for multiple myeloma: a Southwest Oncology Group study. J Clin Oncol 1998:16:890-896.

8. Berenson JR, Crowley JJ, Grogan TM, et al. Maintenance therapy with alternate-day prednisone improves survival in multiple myeloma patients. Blood 2002;99:3163-3168.

9. Offidani M, Corvatta L, Polloni C, et al. Thalidomidedexamethasone versus interferon-alpha-dexamethasone as maintenance treatment after ThaDD induction for multiple myeloma: a prospective, multicentre, randomised study. $\mathrm{Br}$ J Haematol 2008;144:653-659.

10. Ludwig H, Hajek R, Tóthová E, et al. Thalidomide-dexamethasone compared with melphalan-prednisolone in elderly patients with multiple myeloma. Blood 2009;113:3435-3442.

11. Palumbo A, Bringhen S, Rossi D, et al. A prospective, randomized phase III study of bortezomib, melphalan, prednisone and thalidomide (VMPT) versus bortezomib, melphalan and prednisone (VMP) in elderly newly diagnosed myeloma patients [abstract]. Presented at 2008 American Society of Hematology Annual Meeting; December 6-9, 2008; San Francisco, California. Abstract 652.

12. Sonneveld P, van der Holt B, Schmidt-Wolf IGH, et al. First analysis of HOVON-65/GMMG-HD4 randomized phase III trial comparing bortezomib, adriamycine, dexamethasone (PAD) vs $\mathrm{VAD}$ as induction treatment prior to high dose melphalan (HDM) in patients with newly diagnosed multiple myeloma (MM) [abstract]. Presented at the 2008 American Society of Hematology Annual Meeting; December 6-9, 2008; San Francisco, California. Abstract 653. 
13. Barlogie B, Tricot G, Anaissie E, et al. Thalidomide and hematopoietic-cell transplantation for multiple myeloma. $\mathrm{N}$ Engl J Med 2006;354:1021-1030.

14. Attal M, Harousseau JL, Leyvraz S, et al. Maintenance therapy with thalidomide improves survival in patients with multiple myeloma. Blood 2006;108:3289-3294.

15. Abdelkefi A, Ladeb S, Torjman L, et al. Single autologous stem-cell transplantation followed by maintenance therapy with thalidomide is superior to double autologous transplantation in multiple myeloma: results of a multicenter randomized clinical trial. Blood 2008;111:1805-1810.

16. Spencer A, Prince HM, Roberts AW, et al. Consolidation therapy with low-dose thalidomide and prednisolone prolongs the survival of multiple myeloma patients undergoing a single autologous stemcell transplantation procedure. J Clin Oncol 2009;27:1788-1793.

17. Hicks LK, Haynes AE, Reece DE, et al. A meta-analysis and systematic review of thalidomide for patients with previously untreated multiple myeloma. Cancer Treat Rev 2008;34:442-452.

18. Barlogie B, Pineda-Roman $M$, van Rhee $F$, et al. Thalidomide arm of Total Therapy 2 improves complete remission duration and survival in myeloma patients with metaphase cytogenetic abnormalities. Blood 2008;112:3115-3121.

19. Morgan GJ, Jackson GH, Davies FE, et al. Maintenance thalidomide may improve progression free but not overall survival; results from the Myeloma IX Maintenance Randomisation [abstract]. Presented at the 2008 American Society of Hematology Annual Meeting; December 6-9, 2008; San Francisco, California. Abstract 656.

20. Mounier MM, Arnulf B, Jaccard A, et al. Intermittent dex/thal as pretransplantation and maintenance treatment in de novo MM: a randomized trial. Presented at the XIIth International Myeloma Workshop; February 26-March 1, 2009; Washington, DC.

21. Maiolino A, Barros JC, Tanaka $\mathrm{P}$, et al. Thalidomide plus dexamethasone after ASCT improves PFS in MM: a Brazilian randomized trial. Presented at the XIIth International Myeloma Workshop; February 26-March 1, 2009; Washington, DC.

22. Feyler S, Rawstrom A, Jackson G, et al. Thalidomide maintenance following high-dose therapy in multiple myeloma: a UK myeloma forum phase 2 study. Br J Haematol 2007;139:429-433.

23. Chang JE, Juckett MB, Callander NS, et al. Thalidomide maintenance following high-dose melphalan with autologous stem cell support in myeloma. Clin Lymphoma Myeloma 2008;8:153158.

24. Badros A. In the age of novel therapies, what defines high-risk multiple myeloma? J Natl Compr Cancer Netw 2010;8(Suppl 1):S28-S34.

25. Spencer A, Prince M, Roberts AW, et al. First analysis of the Australasian Leukaemia and Lymphoma Group (ALLG) trial of thalidomide and alternate day prednisolone following autologous stem cell transplantation (ASCT) for patients with multiple myeloma (ALLG MM6) [abstract]. Blood 2006;108:Abstract 58. 